\title{
No evidence of association of MUC-1 genetic polymorphism with embryo implantation failure
}

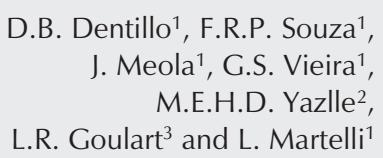

\author{
${ }^{1}$ Departamento de Genética, ${ }^{2}$ Departamento de Ginecologia e Obstetrícia, \\ Faculdade de Medicina de Ribeirão Preto, Universidade de São Paulo, \\ Ribeirão Preto, SP, Brasil \\ ${ }^{3}$ Instituto de Genética e Bioquímica, Universidade Federal de Uberlândia, \\ Uberlândia, MG, Brasil
}

Correspondence

D.B. Dentillo

Departamento de Genética

Bloco C, FMRP, USP

Avenida Bandeirantes, 3900

14049-900 Ribeirão Preto, SP

Brasil

Fax: +55-16-3633-1610

E-mail: danieldentillo@yahoo.com.br

Research supported by CAPES and FAEPA-HCFMRP.

Publication supported by FAPESP. $\ldots \ldots \ldots \ldots \ldots \ldots$

Received May 12, 2006 Accepted April 18, 2007 .........................

\begin{abstract}
Pregnancy loss can be caused by several factors involved in human reproduction. Although up to $50 \%$ of cases remain unexplained, it has been postulated that the major cause of failed pregnancy is an error of embryo implantation. Transmembrane mucin-1 (MUC-1) is a glycoprotein expressed on the endometrial cell surface which acts as a barrier to implantation. The gene that codes for this molecule is composed of a polymorphic tandem repeat of 60 nucleotides. Our objective was to determine if $M U C$ - 1 genetic polymorphism is associated with implantation failure in patients with a history of recurrent abortion. The study was conducted on 10 women aged 25 to 35 years with no history of successful pregnancy and with a diagnosis of infertility. The control group consisted of 32 patients aged 25 to 35 years who had delivered at least two full-term live children and who had no history of abortions or fetal losses. MUC-1 amplicons were obtained by PCR and observed on agarose and polyacrylamide gel after electrophoresis. Statistical analysis showed no significant difference in the number of MUC-1 variable number of tandem repeats between these groups $(\mathrm{P}>0.05)$. Our results suggest that there is no effect of the polymorphic MUC-1 sequence on the implantation failure. However, the data do not exclude MUC-1 relevance during embryo implantation. The process is related to several associated factors such as the mechanisms of gene expression in the uterus, specific MUC-1 post-translational modifications and appropriate interactions with other molecules during embryo implantation.
\end{abstract}

Pregnancy loss has been attributed to several factors involved in human reproduction: genetic and uterine abnormalities, endocrine and immunological dysfunctions, infectious agents, environmental pollutants, psychogenetic factors, and endometriosis (1). Although several medical causes have been
Key words

- Mucin-1

- Implantation failure repeats

- Genetic polymorphism
- Female infertility

- Variable number of tandem established, up to $50 \%$ of cases of recurrent pregnancy loss, defined as two or more spontaneous abortions, still remain unexplained after standard gynecological, hormonal, and karyotype investigations (2). However, it has been postulated that the major single cause of failed pregnancy is an error of embryo 
implantation, which may be as high as $78 \%$ in humans (1).

Embryo implantation is a complex and progressive process which involves synchronous molecular events in the uterus and the embryo promoting a perfect interaction between them. This phenomenon is characterized by apposition, adhesion and invasion of the endometrium by the blastocyst.

The establishment of consistent junctions between the embryo trophoblast and the endometrium requires the expression of adhesion molecules and elimination of nonadhesive molecules from the apical cell lining of both tissues. In this phase, called pre-implantation period, the embryo plays an important role in the receptive phase of implantation, modulating endometrial molecules and controlling the implantation process (3).

Although the details of implantation vary among different species, the basic features of blastocyst attachment to and penetration of the uterine surface epithelium are common to many mammals (4). Based on these data, many experimental animal models have been elaborated to elucidate the main molecular events which occur at the beginning of implantation. One of the molecules that have been extensively studied is mucin-1 (MUC1), also known as episialin (5).

MUC-1 is a glycoprotein expressed on the epithelial surface of different types of tissues, including the endometrium $(6,7)$. This mucin has a polymorphic extracellular protein core containing a large domain of variable number of 20 highly conserved amino acids in tandem repeats, which in humans range from 30 to 90 repeats $(8,9)$.

The presence of MUC- 1 on the endometrial cell surface plays an important role in reproductive genetics because the molecule can act as a barrier, maintaining the uterus non-receptive to the embryo (10).

In 1998, DeLoia et al. (11) observed a great reduction in the expression of MUC-1 in uterine surface epithelium during the receptive phase in mice. The same observa- tions were made in sheep, pigs and baboons, showing that MUC-1 expression is downregulated during the implantation window (12-14).

In humans, although progesterone upregulates MUC-1 expression in the receptive state of the uterus, it is controversial if this molecule has a true anti-adhesion function on the apical epithelial surface of the endometrium or if MUC-1 acts as the first site of embryo attachment (3). However, we cannot exclude the possibility that the embryo has a local effect on the implantation site, cleaving or locally down-regulating MUC-1, as observed in rabbits (15).

Horne et al. (7) compared fertile and infertile women in an attempt to associate the failure of embryo implantation with $M U C-1$ allele size, analyzing the polymorphic variable number of tandem repeats (VNTRs) of the MUC-1 gene region. The results indicated a higher frequency of smaller alleles in women with infertility due to embryo implantation failure when compared to patients with no history of infertility.

In contrast, Goulart et al. (16), comparing the MUC-1 genotype of 10 fertile and 10 infertile women, proposed that there was no association between VNTRs and embryo implantation in women with history of implantation failure or in women with reproductive success.

The aim of the present study was to determine if $M U C-1$ polymorphism is related to implantation failure in women with a history of recurrent abortion and with a diagnosis of infertility.

The infertile group consisted of $10 \mathrm{pa}-$ tients aged from 25 to 35 years, with no history of successful pregnancy, presenting two or more pregnancy losses and a diagnosis of infertility. The patients were evaluated on the basis of routine parameters including a complete hormonal profile (prolactin, free thyroxine, follicle-stimulating hormone, luteinizing hormone, and testosterone) and full- 
cycle evaluation. Tubal patency was confirmed by hysterosalpingography. The results of all tests were within the normal range, and the patients were defined as having unexplained infertility. All of them presented normal karyotypes with 46,XX chromosomes after conventional cytogenetic analysis.

The control group consisted of 31 patients referred to the Contraception Outpatient Clinic of the University Hospital, School of Medicine of Ribeirão Preto, University of São Paulo, SP, Brazil. The inclusion criteria for the fertile group were age from 25 to 35 years, at least two term pregnancies, 2 live children, and no history of abortions or fetal losses.

Approximately $2.0 \mathrm{~mL}$ of peripheral blood was collected from each patient into sterile Vacutainer ${ }^{\circledR}$ tubes containing EDTA. Genomic DNA was extracted by adding 1.0 $\mathrm{mL}$ of lysis buffer $(12 \mathrm{mmol} / \mathrm{L}$ Tris- $\mathrm{HCl}, \mathrm{pH}$ 7.5, $5 \mathrm{mmol} / \mathrm{L}$ EDTA, pH 7.5, $320 \mathrm{mmol} / \mathrm{L}$ sucrose, $5 \mathrm{mmol} / \mathrm{L} \mathrm{MgCl}_{2}$, $4 \%$ Triton $\mathrm{X}$ 100) to $500 \mu \mathrm{L}$ of whole blood. The DNA was dried at room temperature and dissolved in $60 \mu \mathrm{L}$ of sterile ultrapure water.

The desired PCR products flank the VNTR region of the $M U C-1$ gene (GenBank accession No. M61170). They were obtained using the primer pair AGCCACAGCCCCG GTTCAGG (sense, nucleotide position 3627 3646) and GTGCACCAGAGTAGAAGC TGAGCC (antisense, nucleotide position 3999-4022). The amplification conditions were: $20 \mathrm{pmol}$ of primers, $0.75 \mathrm{mmol} / \mathrm{L}$ $\mathrm{MgSO}_{4}, 300 \mu \mathrm{mol} / \mathrm{L}$ dNTP, $0.5 \mathrm{U}$ Pfx DNA polymerase, 1X Pfx buffer, 1X enhancer solution (Invitrogen, Carlsbad, CA, USA), and $50 \mathrm{ng} / \mu \mathrm{L}$ DNA template. Thirty-four cycles were performed according to the following conditions: $35 \mathrm{~s}$ at $94^{\circ} \mathrm{C}, 1 \mathrm{~min}$ at $60^{\circ} \mathrm{C}$, and $2 \mathrm{~min}$ and $30 \mathrm{~s}$ at $68^{\circ} \mathrm{C}$. We also preheated a DNA solution at $94^{\circ} \mathrm{C}$ for $3 \mathrm{~min}$ and, after complete PCR cycling, we performed a final extension of $10 \mathrm{~min}$ at $68^{\circ} \mathrm{C}$.

The amplicons were observed after $1.2 \%$ agarose gel electrophoresis. The gel was stained with ethidium bromide (5 $\mu \mathrm{L} / 100$ $\mathrm{mL}$ gel) at $95 \mathrm{~V}$ for $2 \mathrm{~h}$ and $30 \mathrm{~min}$ and the products were visualized under UV light and photographed with a DC120 Kodak Digital Science camera (Rochester, NY, USA). The base pair size of each amplicon was estimated on the basis of a DNA size ladder (1 kb Plus Ladder, Invitrogen). We confirmed the results using $8.0 \%$ polyacrylamide gel. The number of $M U C-1$ VNTR was calculated using the following formula: $\mathrm{N}-275$ $\mathrm{bp} / 60$, where $\mathrm{N}$ is the amplicon size (in base pairs), $275 \mathrm{bp}$ is the outside region of the $M U C-1$ VNTR and 60 is the number of nucleotides within the core repeats that comprise the $M U C-1$ VNTR (Figure 1).

Thirteen control patients were genotyped as homozygotes and 18 as heterozygotes, and 3 homozygotes and 7 heterozygotes were observed in the infertile group. We detected a prevalence of heterozygotes in both groups. Three different alleles with 20, 22, and 34 repeats were observed only in the infertile group, although this group was not as large as the control group. The average sizes of the upper and lower alleles and the allelic range as well as the allelic frequencies of the patients of both groups are shown in Table 1 .

The mean values of samples with unequal variances were compared by the Student $t$-test (PROC TTEST, Satterthwaite method, Statistical Analysis System, 2004) (17) and no significant difference in the

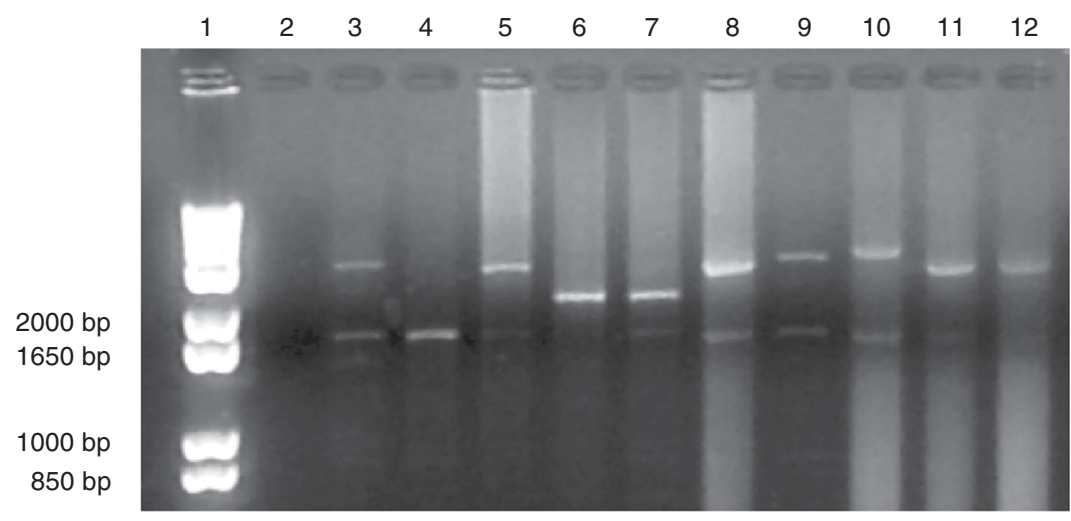

Figure 1. Amplified MUC-1 polymorphic fragments on 1.2\% agarose gel. Lane 1, 1-kb DNA ladder; lane 2, negative control; lanes 3-7, control patients; lanes 8-12, infertile patients. 
number of VNTR motifs was detected between the two groups $(\mathrm{P}>0.05)$. We also excluded the hypothesis that homozygous or heterozygous $M U C$ - 1 VNTR genotype could be related to the fertile or infertile phenotype $(\mathrm{OR}=0.7013, \mathrm{CI}=0.1494-3.2930)$.

Pregnancy loss is one of the leading problems of female health considering that 9 to $13 \%$ of women of reproductive age experience one clinically recognized loss, $5 \%$ experience two or more losses, and 1 to $2 \%$ suffer three or more losses (2), with embryo implantation failure being considered the main problem.

MUC-1 is a high-molecular weight cell surface O-glycosylated-associated glycoprotein expressed in several mammalian cells. In the uterus, the expression of this mucin is

Table 1. Allelic frequency, average size of alleles and allelic range of control and infertile patients.

\begin{tabular}{|c|c|c|}
\hline Allele & $\begin{array}{l}\text { Control group } \\
\qquad(\mathrm{N}=31)\end{array}$ & $\begin{array}{l}\text { Infertile group } \\
\qquad(\mathrm{N}=10)\end{array}$ \\
\hline 14 & 0.016 & - \\
\hline 17 & 0.064 & - \\
\hline 20 & - & 0.100 \\
\hline 22 & - & 0.050 \\
\hline 24 & 0.258 & 0.050 \\
\hline 25 & 0.113 & 0.050 \\
\hline 27 & 0.048 & 0.250 \\
\hline 29 & 0.032 & - \\
\hline 30 & 0.032 & - \\
\hline 32 & 0.048 & - \\
\hline 34 & - & 0.050 \\
\hline 35 & 0.016 & - \\
\hline 39 & 0.032 & 0.050 \\
\hline 45 & 0.032 & - \\
\hline 47 & 0.016 & - \\
\hline 49 & 0.032 & - \\
\hline 52 & 0.016 & - \\
\hline 54 & 0.097 & - \\
\hline 57 & 0.048 & 0.150 \\
\hline 59 & 0.032 & 0.100 \\
\hline 62 & 0.032 & 0.150 \\
\hline Upper allele & $47.87 \pm 14.49$ & $47.2 \pm 16.57$ \\
\hline Lower allele & $28.51 \pm 10.96$ & $32.2 \pm 14.64$ \\
\hline Allelic range & $14-62$ & 20-62 \\
\hline
\end{tabular}

The allele identification numbers correspond to the number of repeats in the $M U C$ - 1 variable number of tandem repeat sequence. Upper and lower allele sizes are reported as means \pm SD. thought to be an important factor in determining endometrial receptivity since it is upregulated during the implantation window (18). Modifications in the internal structure of MUC-1 VNTR could generate changes in the number of core protein O-glycosylation sites, altering molecule immunogenicity (19) and possibly leading to pregnancy loss. Although this is reasonable, the present study indicates that the number of VNTR of $M U C$ $l$ is not associated with implantation failure in women with recurrent abortion.

Horne et al. (7) and Goulart et al. (16) have tried to associate $M U C$ - 1 VNTR polymorphism with female infertility, with discordant results. We could not find additional data about this hypothesis in the literature. The first study found a median size of $3.4 \mathrm{~kb}$ (55 repeats) for the lower allele in the fertile group and $2.5 \mathrm{~kb}$ (40 repeats) in the infertile group, suggesting a higher frequency of smaller $M U C-1$ alleles in women with implantation failure. The second study reported an average size for the lower allele of $1.69 \mathrm{~kb}$ (24 repeats) in both groups. The upper allele presented an average size of $2.49 \mathrm{~kb}$ in the infertile group and $2.35 \mathrm{~kb}$ in the control group. Thus, Goulart and colleagues demonstrated that MUC-1 VNTR polymorphism was not significantly different between the two groups analyzed.

The wide variation among $M U C$ - 1 genotypes in our samples could be related to the multiethnic composition of both groups, in contrast to the study of Horne et al. (7) who investigated a homogeneous sample of 20 European women's genotypes. These investigators considered as fertile, women who delivered at least two full-term children after spontaneous and uneventful pregnancies, but they did not mention if these women had presented any abortions or other gestational history.

In the present study, there was no evidence of an association between the polymorphic VNTR MUC-1 sequence and implantation failure. However, these data are 
based on a small number of selected patients and reflect one of the maternal factors involved in the complex phenomenon of embryo implantation.

\section{Acknowledgments}

We want to express our most grateful thanks to Professors Rui Alberto Ferriani,
Rosana Maria dos Reis and Júlio César Rosa e Silva, Department of Gynecology and Obstetrics, School of Medicine of Ribeirão Preto, who referred the infertile patients to our study. The authors also thank Sílvio Avelino dos Santos for technical assistance and Pedro Alejandro Vozzi for statistical analysis.

\section{References}

1. Bulletti C, Flamigni C, Giacomucci E. Reproductive failure due to spontaneous abortion and recurrent miscarriage. Hum Reprod Update 1996; 2: 118-136.

2. Lissalde-Lavigne G, Cochery-Nouvellon E, Mercier E, Quere I, Dauzat $\mathrm{M}$, Mares $\mathrm{P}$, et al. The association between hereditary thrombophilias and pregnancy loss. Haematologica 2005; 90: 12231230.

3. Pablo JL, Meseguer M, Caballero-Campo P, Pellicer A, Simón C. Embryonic regulation in the process of implantation. In: Gardner D (Editor), Text book of assisted reproductive techniques - laboratory and clinical perspectives. 1st edn. London: Martin Dunitz Ltd.; 2001. p 353-366.

4. Bagchi IC, Li Q, Cheon YP. Role of steroid hormone-regulated genes in implantation. Ann N Y Acad Sci 2001; 943: 68-76.

5. DeSouza MM, Surveyor GA, Price RE, Julian J, Kardon R, Zhou X, et al. MUC1/episialin: a critical barrier in the female reproductive tract. J Reprod Immunol 1999; 45: 127-158.

6. Spicer AP, Parry G, Patton S, Gendler SJ. Molecular cloning and analysis of the mouse homologue of the tumor-associated mucin, MUC1, reveals conservation of potential O-glycosylation sites, transmembrane, and cytoplasmic domains and a loss of minisatellite-like polymorphism. J Biol Chem 1991; 266: 15099-15109.

7. Horne AW, White JO, Margara RA, Williams R, Winston RM, Lalani E. MUC 1: a genetic susceptibility to infertility? Lancet 2001; 357 : 1336-1337.

8. Gendler S, Taylor-Papadimitriou J, Duhig T, Rothbard J, Burchell J. A highly immunogenic region of a human polymorphic epithelial mucin expressed by carcinomas is made up of tandem repeats. $J$ Biol Chem 1988; 263: 12820-12823.

9. Chervenak JL, Illsley NP. Episialin acts as an antiadhesive factor in an in vitro model of human endometrial-blastocyst attachment. Biol Reprod 2000; 63: 294-300.

10. Hey NA, Meseguer M, Simon C, Smorodinsky NI, Wreschner DH,
Ortiz ME, et al. Transmembrane and truncated (SEC) isoforms of MUC1 in the human endometrium and Fallopian tube. Reprod Biol Endocrinol 2003; 1: 2.

11. DeLoia JA, Krasnow JS, Brekosky J, Babaknia A, Julian J, Carson DD. Regional specialization of the cell membrane-associated, polymorphic mucin (MUC1) in human uterine epithelia. Hum Reprod 1998; 13: 2902-2909.

12. Johnson GA, Bazer FW, Jaeger LA, Ka H, Garlow JE, Pfarrer C, et al. Muc-1, integrin, and osteopontin expression during the implantation cascade in sheep. Biol Reprod 2001; 65: 820-828.

13. Bowen JA, Bazer FW, Burghardt RC. Spatial and temporal analyses of integrin and Muc-1 expression in porcine uterine epithelium and trophectoderm in vivo. Biol Reprod 1996; 55: 1098-1106.

14. Hild-Petito S, Fazleabas AT, Julian J, Carson DD. Mucin (Muc-1) expression is differentially regulated in uterine luminal and glandular epithelia of the baboon (Papio anubis). Biol Reprod 1996; 54: 939947.

15. Hoffman LH, Olson GE, Carson DD, Chilton BS. Progesterone and implanting blastocysts regulate Muc1 expression in rabbit uterine epithelium. Endocrinology 1998; 139: 266-271.

16. Goulart LR, Vieira GS, Martelli L, Inacio J, Goulart IM, Franco JG Jr. Is MUC1 polymorphism associated with female infertility? Reprod Biomed Online 2004; 8: 477-482.

17. SAS Institute Inc. SAS OnlineDoc ${ }^{\circledR}$ 9.1.3. Cary: SAS Institute Inc. 2004.

18. Meseguer M, Aplin JD, Caballero-Campo P, O'Connor JE, Martin $\mathrm{JC}$, Remohi J, et al. Human endometrial mucin MUC1 is up-regulated by progesterone and down-regulated in vitro by the human blastocyst. Biol Reprod 2001; 64: 590-601.

19. Santos-Silva F, Fonseca A, Caffrey $T$, Carvalho F, Mesquita $P$, Reis $\mathrm{C}$, et al. Thomsen-Friedenreich antigen expression in gastric carcinomas is associated with MUC1 mucin VNTR polymorphism. Glycobiology 2005; 15: 511-517. 